Manuscript of Nuclear Engineering and Design 2011, 241(6), 1959-1969

DOI: 10.1016/j.nucengdes.2010.09.011

\title{
Determination of the Distribution of Air and Water in Porous Media by Electrical Impedance Tomography and Magneto-Electrical Imaging
}

Franz-Hubert Haegel ${ }^{\mathrm{a}}$, Egon Zimmermann ${ }^{\mathrm{b}}$, Odilia Esser ${ }^{\mathrm{a}}$, Katrin Breede ${ }^{\mathrm{a}}$, Johan Alexander Huisman $^{\mathrm{a}}$, Walter Glaas ${ }^{\mathrm{b}}$, Joachim Berwix ${ }^{\mathrm{b}}$, Harry Vereecken ${ }^{\mathrm{a}}$

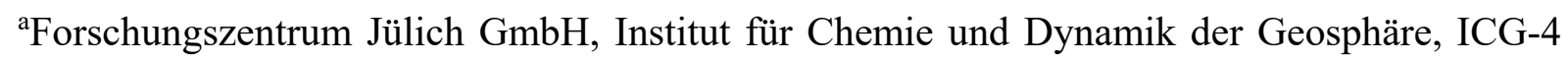
Agrosphäre, 52425 Jülich, Germany

${ }^{\text {b} F o r s c h u n g s z e n t r u m ~ J u ̈ l i c h ~ G m b H, ~ Z e n t r a l i n s t i t u t ~ f u ̈ r ~ E l e k t r o n i k, ~} 52425$ Jülich, Germany

Corresponding author: Franz-Hubert Haegel, phone +49-2461-61-6919, telefax +49-2461-61-2518, e-mail adress f.h.haegel@ff-juelich.de

\begin{abstract}
Monitoring the distribution of water content is essential for understanding hydrological processes in the lithosphere and the pedosphere. The movement of water in unsaturated rock formations and in the vadose zone is influenced by different processes (mainly infiltration, evaporation, percolation and capillary flow) which may be rate determining depending on the actual conditions. The interdependence of these processes also strongly influences the transport and distribution of solutes in the pore space. In order to gain a better understanding of the movement and distribution of water in unsaturated media, systematic investigations with non-invasive or minimal invasive methods appear to be most suitable. Studies on the distribution of electrical conductivity can improve risk analysis concerning waste disposals in general and nuclear waste repositories in particular. Induced polarization and magnetic flux density determined with two highly sensitive accessories yield additional information and may allow for better discrimination of coupled flow processes. Electrical impedance tomography (EIT) with 20 current injection and 48 voltage electrodes was used here to
\end{abstract}


monitor the evaporation of tap water from a container filled with sand under laboratory conditions at $20{ }^{\circ} \mathrm{C}$. The results are compared with data obtained by determining spectral induced polarization (SIP) of sand during desaturation in a multi-step outflow equipment. Infiltration processes and evaporation from sand saturated with $0.01 \mathrm{M} \mathrm{CaCl}_{2}$ were determined by magneto-electrical resistivity imaging technique (MERIT). The results were obtained from a long-term experiment under controlled conditions.

\section{Introduction}

The presence of water strongly influences physical and chemical processes in geological formations. Mechanical and thermal properties as well as dissolution, deposition and transport of materials depend on the water content in the pore space. Therefore knowledge of the spatial and the temporal distribution of moisture in soil and rocks is crucial for understanding and modelling processes in the pedosphere and the lithosphere. Whereas the saturated zone is signified by conditions which are usually near equilibrium, states which are frequently far from equilibrium can be found in the unsaturated pore space. Under these conditions, it is essential for modelling to choose suitable properties controlling the fluxes of water, vapour and gases and to quantify the coupling of different flow mechanisms. Infiltration at the surface or from overlying materials and percolation due to gravity are the processes which govern flow in the saturated pore space. In unsaturated rocks and soil, capillary flow and evaporation are additional transport mechanisms. These four processes largely determine the water content but their interdependence is not yet completely understood. Therefore systematic investigations of the single processes and their coupling are of particular interest in soil science.

The methods and the results presented in this paper are also interesting for the investigation and observation of processes in rock formations which are considered as repositories of nuclear waste. Although disposal in fully saturated plutonic, volcanic, argillaceous, and 
evaporative rocks is usually preferred (Sumerling and Smith, 1998), evaporation and capillary flow play a role for risk analysis in nuclear waste management. Since high level nuclear waste develops heat and produces gases, the pore space near the waste may become unsaturated. In addition, unsaturated tuff is also considered for nuclear waste disposal (e.g. at Yucca Mountain, Nevada, USA). In this case evaporation and capillary effects have to be considered (Bodvarsson et al., 1999).

Several non-destructive imaging techniques have been developed and used for monitoring water in the pore space of consolidated and unconsolidated geological materials (Rubin and Hubbard, 2005). For application in the field, electrical or electromagnetic techniques are most suitable, because the equipment needed is mobile and relatively cheap. There is also a long tradition of electrical methods in the geosciences from ore and oil prospection, and thus ample basic knowledge exists on the use of such instruments and measurement strategies. For the imaging of processes with high spatial resolution, however, the techniques must still be improved in terms of speed and accuracy. Elucidating the relationship between hydrological parameters and electrical parameters is still a matter of current research. Since electrical signals also depend on the properties of matrix and fluid, results obtained in the field are ambiguous in many cases. The database must therefore be enlarged by systematic investigations in the laboratory and a combination of different techniques.

The majority of imaging experiments with electrical techniques in the geosciences has been performed with electrical resistivity tomography (ERT). Recently, the influence of the heterogeneity of soil on the percolation in the vadose zone has been successfully investigated in lysimeter studies (Koestel et al., 2009). The results have also been discussed using a stream tube model (Koestel et al., 2008) as applied for the description of the heterogeneity of an aquifer at field scale (Müller et al., 2010). There are also several papers dealing with investigations on rock formations considered or used for nuclear waste disposal. Yaramanci and Flach (1992) used ERT at the Asse salt mine (Germany) to image the distribution of 
electrical resistivity. The results were used for the evaluation of porosity and water content in rock salt and adjacent anhydrite layers (Yaramanci, 2000). Brine inflow into a transport drift of Asse was also detected by ERT (Yaramanci, 1994). Jockwer et al. (2007) could demonstrate with ERT measurements in a heater experiment at the Mont Terri underground rock laboratory in Switzerland that the desaturation of Opalinus clay could be excluded.

Electrical impedance tomography (EIT) is an extension of ERT considering the phase shift between voltage and current caused by induced polarization (IP). In porous media, electrical conductance is due to different mechanisms. Water in the pore space of soil usually contains considerable concentrations of various ions and exhibits electrical conductivities in the range of $0.01-0.1 \mathrm{~S} \mathrm{~m}^{-1}$. Electrical conductance in water saturated soil is predominantly due to the migration of bulk ions in the electrical field applied by electrodes brought into contact with the soil. There is also a distinct contribution to ohmic conductivity made by ions in the electrical double layer (EDL). These ions compensate charges at the surface of the solid matrix and behave different from bulk ions, because they are influenced by the electrical field of the surface charges. The importance of this surface conductivity increases with decreasing water content. In addition, the distribution of ions in the electrical double layer changes when electrical fields are applied. This gives rise to IP which can be measured either in time (Titov et al., 2004) or frequency domain (Ulrich and Slater, 2004). The latter method is called spectral induced polarisation (SIP). Due to the compression of the electrical double layer with increasing ion concentration, the IP effect dies away with increasing electrolyte concentration. For typical salt concentrations found in soil water, IP is well detectable. In rocks, the effect is usually larger. The measured complex electrical conductivity $\sigma^{*}$ can be expressed as the sum of a real part $\sigma^{\prime}$ and an imaginary part $\sigma^{\prime \prime}$.

$$
\sigma^{*}=\sigma^{\prime}+\mathrm{i} \sigma^{\prime \prime}
$$

Whereas ohmic conductivity $\sigma^{\prime}$ decreases continuously with decreasing water content in sand, $\sigma^{\prime \prime}$ exhibits a maximum at moderate desaturation for the displacement of water by pressure 
(Ulrich and Slater, 2004). For evaporation from sand, a continuous decrease was found for $\sigma "$ by Ulrich and Slater (2004) with SIP. The material properties determined by such experiments may be used for calibrating EIT.

The IP effect yields additional information on the pore structure, the pore size, the water saturation, the chemistry of pore water, and the chemistry of the surface. Kulenkampff and Yaramanci (1993) determined IP on rock salt from Asse and obtained reasonably large effects depending on the frequency of $\mathrm{AC}$ current, but field tests at Asse yielded ambiguous results (Yaramanci, 2000). Kruschwitz and Yaramanci (2004), however, successfully characterized the excavation damaged zone in the Opalinus claystone of Mont Terri with EIT and could distinguish strained and compressed zones by determining the changes of phase angles around a drift. Cosenza et al. (2007) determined the effect of heating on argillite from Tournemire (France) by impedance measurements at low frequencies and found cracking of the argillaceous rock at elevated temperatures in the laboratory. Roberts and Lin (1997) characterized Topopah Spring tuff, which is the host rock at Yucca Mountain, with impedance spectroscopy and found three different regimes of complex electrical conductivity depending on saturation. From their results they could conclude on the structure of the pore space and the cation exchange capacity.

Moving charges are always connected with the induction of a magnetic field, and thus electrical conductivity can also be determined by magnetic measurements. The magnetic flux density at position vector $r$ can be expressed as the integral of contributions of the vector product of gradients of the potential $\mathrm{V}$ and the conductivity $\sigma$ over all volume elements located at position vectors $\mathrm{r}^{\prime}$ according to a modified equation based on the Biot-Savart law (Edwards and Nabighian, 1991).

$$
B(\mathbf{r})=\frac{\mu}{4 \pi} \int_{\mathrm{v}} \frac{\nabla^{\prime} V\left(\mathbf{r}^{\prime}\right) \times \nabla^{\prime} \sigma\left(\mathbf{r}^{\prime}\right)}{\left|\mathbf{r}-\mathbf{r}^{\prime}\right|} d v^{\prime}
$$


The method of measuring magnetic fields for the determination of electrical conductivity is called magnetometric resistivity (MMR) (Nabighian, 1988; Nabighian, 1991). It may be combined with ERT in order to improve the quality of data. Since the symmetry of the magnetic field is different from that of the electric field and the magnetic signal is sensitive to the electric field and the conductivity gradients (see eq. 2), improved images are expected for magneto-electrical resistivity imaging technique (MERIT) (Verweerd, 2007; Rubin and Hubbard, 2005), which combines EIT and MMR. In particular, zones of reduced electrical conductance surrounded by zones of higher electrical conductance may be detected much better by magnetic measurements. The gradients are large at the border between both zones and therefore a considerable magnetic field is expected. EIT will have problems to accurately image the low-conductivity zone because it preferentially samples the zones with higher conductivity. As the magnetic field increases with increasing current and the magnetic fields are rather low, this method is particularly suitable for porous bodies with elevated electrical conductivity. Thus, EIT and MERIT are methods that complement one another.

In this study EIT and MERIT were tested for the investigation of evaporation from sand. Evaporation occurs at the gas/liquid interface. If the adjacent gas phase at the surface or in the unsaturated pore space is not saturated with water vapour, water evaporates from the liquid phase and consequently induces capillary flow. As vapour pressure is strongly dependent on temperature, temperature gradients and heat flow have an influence on the distribution of moisture in unsaturated rocks or soil. Evaporation rates are also strongly influenced by the morphology and interfacial properties of the solid matrix. The extent of the interface between gas and water in a partially saturated pore space may vary widely depending on the absolute size of pore necks and pore bodies as well as on their ratio. Upward flow caused by capillary forces is also strongly dependent on the capillary radius and the wetting properties of the pore space (Shokri et al., 2009). Thus, the distribution and change of moisture content in rocks and soil is a very complex problem, and more detailed knowledge on the elementary processes is 
urgently needed to model its influence on transport processes or weather and climate, as well as on agricultural and irrigation management (Vereecken et al., 2008).

\section{Materials and Methods}

All experiments were performed under controlled temperature conditions at $20{ }^{\circ} \mathrm{C}$ with an accuracy of $\pm 1{ }^{\circ} \mathrm{C}$ in an air-conditioned room. Air humidity was also relatively stable at about $50 \pm 10 \%$.

\subsection{Materials}

Two different sands were used for the experiments, types F 32 and F 34 from Quarzwerke Frechen (Germany). Both sands were obtained from the same source by processing and sorting. They were similar with a somewhat different grain size distribution with average grain sizes of $0.24 \mathrm{~mm}$ (F 32) and $0.20 \mathrm{~mm}$ (F 34). Tap water from Forschungszentrum Jülich was used for experiments with EIT and SIP. It had a conductivity of $0.0450 \mathrm{~S} \mathrm{~m}^{-1}$ at $20{ }^{\circ} \mathrm{C}$. The chemical composition is constantly controlled and variations in the ion concentrations are very small. $0.01 \mathrm{M}$ aqueous $\mathrm{CaCl}_{2}$ was used for the MERIT experiment as the liquid phase. It was prepared with $\mathrm{CaCl}_{2} \cdot 2 \mathrm{H}_{2} \mathrm{O}$ (p.a. from Merck KGaA, Darmstadt, Germany), and ultrapure water. The conductivity of the electrolyte was $0.2080 \mathrm{~S} \mathrm{~m}^{-1}$ at $20{ }^{\circ} \mathrm{C}$.

\subsection{Equipment}

\subsubsection{MERIT}

Electric field and magnetic flux density values were simultaneously determined in a 10-1 DURAN glass beaker with an inner height of $342 \mathrm{~mm}$ and inner diameter of $210 \mathrm{~mm}$. Current was injected with a frequency of $25 \mathrm{~Hz}$ at the central axis (z-axis) of the beaker by 8 annular electrodes with an outer diameter of $8 \mathrm{~mm}$ and height of $5 \mathrm{~mm}$, fixed on a printed board with a length of $290 \mathrm{~mm}$ and width of $4 \mathrm{~mm}$. The distance between the electrodes was $30 \mathrm{~mm}$. The 
position of the printed board was fixed by two tripods at the top and bottom of the beaker at $27 \mathrm{~mm}$ and $276 \mathrm{~mm}$ above the inner surface of the bottom of the beaker. The position of this inner surface of the beaker is referred to as $z=0$. The centre of the uppermost electrode (electrode 8) was placed at $\mathrm{z}=252 \mathrm{~mm}$. The experimental assembly is schematically shown in Figure 1.

The three components of magnetic flux densities in the direction of the z-axis, as well as in the tangential and radial direction were measured with 24 sets of AMR sensors situated at a distance of $140.6 \mathrm{~mm}$ (for the tangential component) and $148.4 \mathrm{~mm}$ (for the other components) from the central axis on a movable scanner ring surrounding the beaker symmetrically with respect to the z-axis. The scanner and the sensors are described in detail in Zimmermann et al. (2005). A $25^{\text {th }}$ set of sensors was placed about $565 \mathrm{~mm}$ from the centre of the beaker at $\mathrm{z} \approx 230 \mathrm{~mm}$ in order to detect fluctuations of the $25 \mathrm{~Hz}$ component of the outer magnetic field.

\subsubsection{SIP}

A laboratory set-up was developed to investigate the relationship between the hydraulic and electrical properties of unsaturated porous media. The design is similar to Ulrich and Slater (2004). In summary, pressure is applied to a PMMA sample holder of $100 \mathrm{~mm}$ height and an inner diameter of $80 \mathrm{~mm}$ filled with sand. The resulting water outflow is automatically recorded and can be used to calculate the volumetric water content of the soil sample if the soil material is dried after completion of the experiment. SIP measurements were made using the equipment and design considerations presented in Zimmermann et al. $\left(2008^{\mathrm{a}}\right)$.

\subsubsection{EIT}

Electrical impedance tomography was performed in a cylindrical container made from PMMA with a height of $363 \mathrm{~mm}$ and an inner diameter of $280 \mathrm{~mm}$. The container was 
equipped with 4 rows of 17 bores each for cylindrical brass electrodes with a diameter of 6 mm which were used for current injection and potential measurements. Each row had 5 current and 12 voltage electrodes. The electrodes were located 62, 122, 182, $242 \mathrm{~mm}$ above the inner surface of the bottom of the container which is defined as position $z=0$. Data were collected by a system described in detail by Zimmermann et al. $\left(2008^{\text {b }}\right)$.

\subsection{Procedures}

\subsubsection{MERIT}

$200 \mathrm{ml}$ of $0.01 \mathrm{M} \mathrm{CaCl}_{2}$ solution were placed in the 10-1 glass beaker. Small portions of about $600 \mathrm{ml}$ of $\mathrm{F} 34$ sand were mixed into $300 \mathrm{ml}$ of $\mathrm{CaCl}_{2}$ solution and the mixture was poured into the 10-1 beaker. This procedure was repeated until the beaker was filled with saturated sand up to $13 \pm 3 \mathrm{~mm}$ above the centre of the uppermost electrode. Initially, the surface was located at $\mathrm{z}=265 \mathrm{~mm}$. The position changed slightly during desaturation to $\mathrm{z}=263 \mathrm{~mm}$. The supernatant $\mathrm{CaCl}_{2}$ solution (about $200 \mathrm{ml}$ ) was removed with a pipette and the reference values were determined at 6 different equidistant $(50 \mathrm{~mm}$ ) positions (for the tangential component) between $\mathrm{z}=40 \mathrm{~mm}$ and $\mathrm{z}=290 \mathrm{~mm}$ with 16 different independent electrode configurations and the reverse configurations for current injection. Swapping the current injection led to different results owing to offsets caused by the electronic components. At each position and for each current injection two measurements were performed. Magnetic flux density and voltage data were collected for $10 \mathrm{~s}$. The time needed for the whole scan was 65 min. Further reference measurements for statistical evaluation of magnetic flux density data were performed at sensor positions $\mathrm{z}=150 \mathrm{~mm}$ and $\mathrm{z}=240 \mathrm{~mm}$. In these cases, 20 measurements were performed for each of two different current injections and the reverse configurations of the current electrodes. Voltages were not evaluated for these measurements. The time needed for these measurements was $25 \mathrm{~min}$. The voltage applied for current injection was $100 \mathrm{~V}(\mathrm{p} / \mathrm{p})$. 
$120 \mathrm{ml}$ of ultrapure water with a conductivity of $2 \cdot 10^{-5} \mathrm{~S} \mathrm{~m}^{-1}$ was infiltrated into the sand with a funnel at positions $\mathrm{r}=50 \mathrm{~mm}, \phi=52.5^{\circ}$ and $\mathrm{z}=90 \mathrm{~mm}$, where $\mathrm{r}$ is the distance from the central axis of the beaker, $\phi$ the angle with respect to the direction of sensor 1 (positive $\mathrm{x}$ axis), and $\mathrm{z}$ the position of the end of the tube of the funnel placed in the sand. The tube of the funnel had an inner diameter of $3 \mathrm{~mm}$. After infiltration of the water, which took about 3 hours, a scan was performed with the same parameters as the reference scan. Thereafter, the sand in the beaker was left for about 6 months under controlled laboratory conditions $\left(20{ }^{\circ} \mathrm{C}\right.$ and $50 \pm 10 \%$ air humidity) for evaporation of the water. Consecutive measurements were performed during this period. After the evaporation experiment, $120 \mathrm{ml}$ of $1 \mathrm{M} \mathrm{CaCl}_{2}$ solution with a conductivity of $13.35 \mathrm{~S} \mathrm{~m}^{-1}$ was infiltrated into the dry sand with a funnel at positions $\mathrm{r}$ $=65 \mathrm{~mm}, \phi=270^{\circ}$ and $\mathrm{z}=95 \mathrm{~mm}$.

\subsubsection{SIP}

Initially, a layer of degassed tap water was filled into the sample holder. Next, F34 sand was added and allowed to settle. This procedure was repeated until the sample holder was filled. The porosity of the sample was approximately $38 \%$ using this procedure. Eight pressure steps were applied (10, 40, 50, 52, 56 60, 65, 70 mbar) and outflow was monitored. When the electrical signal was constant for $24 \mathrm{~h}$ and the outflow was very low, the next pressure step was applied. The measured outflow was used to determine the volumetric water content and SIP measurements were made at a range of frequencies from $1 \mathrm{mHz}$ to $45 \mathrm{kHz}$, but here we only consider the complex conductivity at $1 \mathrm{~Hz}$.

\subsubsection{EIT}

Another evaporation experiment was performed with EIT in a cylindrical container. Initially, about 5.51 of degassed tap water was poured into the empty container until the water level was approximately in the centre between the lower two electrode rows. The air in the bores 
for the electrodes was removed in the lowest row of electrodes by injecting water with a wash bottle. Then dry F 32 sand was filled into the container in three portions until the sand surface was located in the middle between the two lowest electrode rows (rows 1 and 2). The sand was compacted by rocking the container after each addition. The water level rose to the middle between the electrode rows 2 and 3. Subsequently, the air in the bores was removed in the electrode row 2. Then, two portions of sand were filled into the container and compacted by rocking. Thereafter, the water level was situated between the two uppermost electrodes (rows 3 and 4). The air from the bores in electrode row 3 was removed, and another two portions of sand were filled into the container and compacted by rocking. Two cylinders were placed in the sand, one made of aluminium and one of PVC, for test reasons. Sand was then added in small quantities until the water level rose above the bores in the uppermost electrode row (row 4). $250 \mathrm{ml}$ of degassed tap water were added and the air was removed from the bores in row 4. Finally, sand was filled into the container in small quantities and compacted by rocking until the desired position of the sand surface was obtained. The supernatant was removed by a syringe. Both cylinders were removed from the container after a test measurement, which will not be discussed here. The packing of the sand was visibly disturbed at those locations where the objects had been. Some larger air bubbles were observed here. Only some of the air could be removed by rocking the container; some smaller bubbles remained in the sand. The initial level of the sand was at $\mathrm{z}=303 \pm 3 \mathrm{~mm}$. The level reduced to $\mathrm{z}=296 \pm 3 \mathrm{~mm}$ during evaporation. A reference measurement was made immediately after removing the test bodies and rocking the container. Subsequent measurements were performed during evaporation for about 5 months. For each measurement, data for 20 different current injections and the 20 reverse injections were determined. Voltage was measured with 48 electrodes simultaneously versus ground. Spectra of induced polarization were collected at 14 frequencies between $1 \mathrm{~Hz}$ and $10 \mathrm{KHz}$ for each current injection. The time needed for one complete measurement was $30 \mathrm{~min}$. 


\subsection{Imaging}

A regularized iterative reconstruction routine programmed in MATLAB was used for the imaging of EIT and MERIT. The inverse problem was solved as an optimization problem. At each iteration, starting from a homogeneous distribution of electrical conductivity $\sigma_{0}$, an objective function consisting of the data misfit and a regularization term (here the roughness of the distribution) was minimized (deGroot-Hedlin and Constable, 1990). The data misfit is the difference between the measured sensor signals and the theoretical sensor signals. In order to calculate the theoretical signals, 3D modelling based on the finite element method was used, employing an irregular mesh of tetrahedrons (Kwon and Bang, 1997; Xu et al., 2005). This method was used to solve the Poisson equation (Equation 3)

$$
\nabla \cdot\left[\sigma^{*}(\mathbf{r}) \nabla V(\mathbf{r})\right]=-I \delta(\mathbf{r})
$$

which describes the relation between the electrical potential $\mathrm{V}$ in the medium with the complex conductivity distribution $\sigma^{*}$ and a quasi static point-source current injection I. $\delta$ denotes the Dirac delta function. The potential differences between the voltage electrodes, normalized on the injected current, were calculated in the case of EIT, yielding the corresponding theoretical sensor signals (Zimmermann et al., 2008 ${ }^{\mathrm{b}}$ ).

In the case of MERIT, the electrical signal was used for 1D inversion, because the chosen electrode configuration is only sensitive to changes in the z-direction. It is indeterminate with respect to the direction $\phi$ (angle with the positive $\mathrm{x}$-axis) due to the radial symmetry of the annular electrodes arranged on the central axis of the beaker (z-axis). The radial resolution is rather low, because most of the current flows near the electrodes and not through the major volume of the sand.

The forward modelling is done in 3D with a mesh of tetrahedrons for the conductivity elements that does not predetermine a special horizontal layering. In order to determine the 
1D conductivity distribution, the inversion routine only allowed changes of the conductivity in $\mathrm{z}$-direction.

Whereas the lower boundary of the sand body was well defined by the bottom of the container, the surface was not exactly flat. This caused erroneous inhomogeneous images for the saturated sand, when the complete data set was used. Because the uppermost electrode (electrode 8$)$ was very close to the surface $(13 \mathrm{~mm})$, small changes of the position of the surface level resulted in relatively large variations of the inverted 1D conductivity distribution. It is suspected that this reconstruction error is caused by the roughness of the surface of about $3 \mathrm{~mm}$ and the high sensitivity near the current injection electrodes. To remove this reconstruction error, the electrical data obtained for current injections from the uppermost electrode were omitted. The other current injections were considerably less affected by surface roughness and yielded satisfying results.

For MMR images, the magnetic flux density at the position of the sensors must be calculated first. It is the superposition of the magnetic flux density induced by the current density $\mathrm{J}$ in the medium

$$
J(\mathbf{r})=-\sigma(\mathbf{r}) \nabla V(\mathbf{r})
$$

and the magnetic flux density induced from the current in the cables. In order to compute the magnetic flux density, the Biot-Savart law was used in the usual form

$$
B(r)=\frac{\mu_{0}}{4 \pi} \int_{v} \frac{J\left(\mathbf{r}^{\prime}\right) \times\left(\mathbf{r}-\mathbf{r}^{\prime}\right)}{\left|\mathbf{r}-\mathbf{r}^{\prime}\right|^{3}} d v^{\prime}
$$

Finally, the magnetic flux density at the sensor position yields the theoretical sensor signal which will be measured (Zimmermann et al., 2005). At present, the electrical and the magnetic signals are used only separately for MERIT imaging. Joint imaging is still in preparation. For 2D respectively 2.5D imaging see also Kemna et al. (2003). 


\section{Results and Discussion}

\subsection{MERIT}

\subsubsection{Statistical evaluation of magnetic flux density}

Three components of magnetic flux density were measured. $\mathrm{B}_{\mathrm{z}}$ which is the component in the direction of the symmetry axis of the measurement setup was rather low and did not yield good results. The values for all 24 sensors of the scanner were only somewhat larger than the values obtained for sensor 25 which was located away from the probe. Since the components of the electrical field for the central current injection are mainly in direction of the symmetry axis of the measurement setup, this result was not surprising. The radial component $\mathrm{B}_{\mathrm{r}}$ and the tangential component $\mathrm{B}_{\mathrm{t}}$ showed distinct signals which varied with time during the evaporation of water from F 34 sand. Figure 2 a shows typical curves for one current injection (electrodes 7 and 2) and the reverse current injection (electrodes 2 and 7) for the difference $\Delta \mathrm{B}_{\mathrm{t}}$ at sensor 1 compared to the reference measurement. For comparison, one of the data sets for sensor 25 (electrodes 2 and 7) is also included in the figure. It is clearly visible that the absolute values of magnetic flux densities in the sand first increase and then decrease with time. The maximum absolute values were obtained for most sensors and components $\Delta \mathrm{B}_{\mathrm{r}}$ and $\Delta \mathrm{B}_{\mathrm{t}}$ after about 14 days in the upper part of the sand in the beaker at $\mathrm{z}=240 \mathrm{~mm}$.

Furthermore, it can be seen from Figure $2 \mathrm{a}$ that the values of $\Delta \mathrm{B}_{\mathrm{t}}$ do not relax to 0 , but end at a certain asymptotic value. This behaviour reflects the phenomena observed during evaporation from outside the glass beaker. Strata of dark and pale sand were observed during evaporation. This means that the inhomogeneity of the water content in the sand increased in relation to the complete saturation at the beginning. Since the current injected decreased during evaporation (Figure 2b), $\Delta \mathrm{B}_{\mathrm{t}}$ must increase with time for an inhomogeneous distribution of electrical conductivities. For a homogeneous distribution of electrical conductivities in the sand, there should be no signal of $\Delta \mathrm{B}_{\mathrm{t}}$ for central current injection. 
The curve for the current $\mathrm{I}$ in Figure $2 \mathrm{~b}$ reflects the loss of water and the reduction of the electrical conductivity with time. The curve of $\Delta \mathrm{B}_{\mathrm{t}}$ can be explained by the medium becoming inhomogeneous during evaporation and retaining some of the inhomogeneity after drying. The resulting difference in electrolyte content at different locations and times led to an inhomogeneity of ion distribution in the sand and thus to an inhomogeneity of surface conductivity which is still observed in the dry sand with $\Delta \mathrm{B}_{\mathrm{t}}$ being different from 0 .

\subsubsection{Imaging}

Both the electrical (EIT) as well as the magnetic component (MMR) of the MERIT measurements were used for imaging the conductivity distribution in the sand for a long-term experiment on evaporation and two infiltration experiments. Prior to evaporation ultrapure water was infiltrated as a negative tracer into the sand saturated with $0.01 \mathrm{M} \mathrm{CaCl}_{2}$ solution $90 \mathrm{~mm}$ below the surface. After the evaporation experiment a salt tracer $\left(1 \mathrm{M} \mathrm{CaCl}_{2}\right)$ was infiltrated into the dry sand $95 \mathrm{~mm}$ below the surface. Prior to the first infiltration the conductivity distribution determined by EIT in the saturated sand was rather homogeneous (Figure 3) taking into account that the image is reconstructed from a very limited data set of 14 current injections obtained with 7 electrodes. The lower value at the bottom of about 0.04 $\mathrm{S} \mathrm{m}^{-1}(\mathrm{z}=5 \mathrm{~mm})$ is due to the tripod of PMMA installed in the sand for centring the board with the electrodes. The other values are between 0.045 and $0.055 \mathrm{~S} \mathrm{~m}^{-1}$. The patterns appearing in some horizontal sections are a consequence of the irregular tetrahedral mesh that was used for 3D forward modelling.

Figure $4 \mathrm{a}$ shows the conductivity obtained by $1 \mathrm{D}$ inversion of EIT in z-direction immediately after the infiltration of the negative tracer. The conductivities at $160 \mathrm{~mm}$ and lower are slightly decreased compared with the conductivities in Figure 3. At the top, conductivity increased due to the displacement of electrolyte into a thin liquid layer with considerably higher conductivity. Obviously this effect also influences the result at $\mathrm{z}=212 \mathrm{~mm}$. The 
reduction of the conductivity by the negative tracer is much better detected from the 3D MMR image (Figure 4b). Whereas EIT yields absolute values of conductivity, MMR shows relative values of the differences of conductivity referred to a reference state. The reference for the infiltration of the negative tracer and the subsequent evaporation was determined just prior to the infiltration. The height and the direction of the decrease in relative conductivity (dark area) in Figure $4 \mathrm{~b}$ is correct. The distance from the centre, however, could not be exactly reconstructed. There are also smaller zones of weaker positive and negative changes in the image which are obviously artefacts. Nevertheless, the results of the negative tracer infiltration demonstrate that MMR is a useful method for the detection of low conductivities in a matrix of high conductivity. This situation is often found at contaminated sites with nonaqueous phase liquids and can hardly be resolved by EIT alone. The method can be improved with electrical data from electrodes which are located near the magnetic sensors and joint inversion with magnetic data. It will be particularly interesting for monitoring in environments with elevated conductivity as found in zones of irrigation, near the coast, or in saline rock formations used for $\mathrm{CO}_{2}$ storage (see e.g. Giese et al., 2009).

In contrast to the results obtained for infiltration, EIT provides the more pronounced data for evaporation. Whereas the reduction of conductivity at the top of the container after 9 days is clearly visible in Figure 5a, the contrast of the MMR inversion is much weaker (Figure 5b). There, however, the remains of the negative tracer and some additional inhomogeneities at the bottom are visible. There is now also a reduction of conductivity in the direction of the negative tracer infiltration at $\mathrm{z}=212 \mathrm{~mm}$ which is due to an upward flow of the negative tracer caused by buoyancy forces and capillary forces due to evaporation. The inhomogeneity found at the bottom might be an artefact, but a similar effect was also found for a similar experiment studied with EIT which will be discussed later on.

After about 6 month the evaporation experiment was finished and a salt tracer was infiltrated into the dry column. The increase in conductivity could not be observed with EIT, because the 
absolute change was too low. 3D MMR inversion referred to the dry state before infiltration, however, showed a large increase of conductivity in direction of the negative y-axis, were the salt tracer had been infiltrated (Figure 6). Again the height and the direction of the tracer are correctly reconstructed, but the distance from the centre is not correct. This effect is mainly due to the fact that inversion is an ill-posed problem and the measured data can be better fit with a larger number of voxels available in a certain direction as found at the periphery of the cylinder. Nevertheless, this result on the infiltration of a salt tracer into a matrix of very low conductivity is particularly interesting for monitoring in rock salt formations, for example, to identify and locate thin layers of anhydrite as found by Kessels et al. (1985) in the Asse salt dome or for early detection of leaks.

\subsection{SIP}

Since evaporation is a very complex process as shown by the MERIT experiment, another method was used for investigating the relationship between the hydraulic and electrical properties during desaturation of F 34 sand. Spectral induced polarization was determined during a multi-step outflow experiment. In this case, water is removed from the pore space by pressure. An inhomogeneous distribution of the electrolyte concentration in the liquid phase should not occur or only to a much lesser extent, if subsequent redistribution processes are involved. The results of this experiment are shown in Figures $7 \mathrm{a}$ and $\mathrm{b}$. Data are presented in the same way as done by Ulrich and Slater (2004). The values of the real part of conductivity $\sigma^{\prime}$ and the imaginary part $\sigma^{\prime \prime}$ are normalized to the values $\sigma^{\prime} 0$ and $\sigma^{\prime \prime} 0$ obtained for the saturated sand at the beginning of the experiment. The corresponding normalized values are denoted as $\mathrm{S}^{\prime}$ and $\mathrm{S}^{\prime \prime}$. The values of $\sigma^{\prime} 0$ and $\sigma^{\prime \prime} 0$ were $1.22 \cdot 10^{-2} \mathrm{~S} \mathrm{~m}^{-1}$ and $1.1 \cdot 10^{-4} \mathrm{~S} \mathrm{~m}^{-1}$. The volumetric water content of the sand was initially $38 \%$. The water saturation $\mathrm{S}_{\mathrm{w}}$ is the volumetric water content normalized to this initial value for the completely saturated sand. The results are similar to those obtained by Ulrich and Slater (2004) for similar sand (sample 
$\mathrm{G}$ in that paper). Whereas the real part of the electrical conductivity decreases continuously with decreasing water saturation, the imaginary part shows a maximum at moderate desaturation. Since the real part and the imaginary part of the electrical conductivity are usually not linearly dependent from each other, as shown by different slopes in the double logarithmic plots, the additional determination of IP yields additional information for imaging. The imaginary part of the electrical conductivity is a more complex function of water saturation in the case of pressure-induced desaturation, in particular, and thus may be also useful for monitoring pressure filtration.

\subsection{EIT}

A similar evaporation experiment as already presented for MERIT was performed in a larger container and observed by EIT. The phenomena which were visible from outside were similar to those observed during the MERIT experiment. The distribution of conductivities $\sigma^{\prime}$ and $\sigma^{\prime \prime}$ for $1 \mathrm{~Hz}$ before the experiment began, and after 7 and 21 days is shown in Figures 8a-c. Despite some erroneous values obtained near the electrodes, the real part of conductivity (ohmic conductivity) was constant in the entire sand body and the imaginary part was more than three orders of magnitude lower at the beginning. Elevated values of $\sigma$ " were caused by disturbed locations in the sand which were described in section 2.3.2.. With progressive desaturation, these large values disappeared. However, errors found near some electrodes were also so large, that the effects of desaturation on the imaginary part could not be unequivocally detected. After 21 days, increased values of $\sigma$ " were found at the bottom in the centre of the container near a zone of enhanced conductivity $\sigma^{\prime}$. This zone is formed during the experiment. Whereas after 7 days (Figure 5b), only reduced values of $\sigma$ ' were found in the upper part of the sand and to a much smaller extent at the bottom, the formation of a core zone of enhanced conductivity in the lower part of the container is clearly visible after 21 days (Figure 8c). The enhanced imaginary conductivities found there are still not yet well 
understood, but they correspond to observations of unusually high values often found in the field compared to laboratory experiments.

The effect of evaporation on the overall conductivity distribution and the core formation can also be seen in Figure 9. The percentage of voxels with conductivity larger than the values of $\sigma^{\prime}$ on the abscissa is plotted here in a cumulative representation. The conductivity continuously decreases with time, but after 21 days some percent of voxels with enhanced conductivity appear. This effect is the result of different processes. On the one hand evaporation at the drying front in the upper part of the container causes capillary flow from the lower parts. On the other hand, the electrolyte concentration increases in the remaining water at the drying front leading to density gradients and gradients of osmotic pressure. The effect is obviously not one-dimensional. Wall effects are observed. The reduction of conductivity at the bottom near the wall after 21 days (Figure 10 a) was also clearly visible from outside. At the bottom, a zone was observed which was drier than the overlying lower part of the container at this time. For the wall effects, thermal gradients are to be considered, but convection which is due to gradients of capillary pressure, density and osmotic pressure might also be disturbed at the wall. The effect is still present 4 months later (Figure $10 \mathrm{~b}$ ) when the overall conductivity is already one order of magnitude lower. The largest values of conductivity are now found at the bottom of the container in accordance with progressive evaporation and density flow.

The enhanced $\sigma^{\prime \prime}$ values beneath the zone of high $\sigma^{\prime}$ (Figure 8c) may be attributed to the desaturation at the bottom which was visually observed at this time. The values of up to $8 \cdot 10^{-4}$ $\mathrm{S} \mathrm{m}^{-1}$, however, are considerably higher than those found by SIP measurements on pressureassisted desaturation. This result is not in accordance with the findings in the paper of Ulrich and Slater (2004), but the design of the evaporation experiment performed there, the sand, and the electrolyte concentration were different. The properties of the sand and the considerably higher electrolyte concentration used in the EIT experiment should not be responsible for the 
enhanced imaginary part of electrical conductivity found at the bottom of the container. However, the samples of Ulrich and Slater (2004) were much smaller and evaporation was performed by opening both ends of the column leading probably to much better homogeneity of the sample during evaporation.

\section{Conclusion}

With MERIT and EIT, additional data are collected with respect to ERT measurements alone. They can be used to gather additional information about processes observed during the desaturation of porous media and for enhanced imaging of soil moisture distribution. Both methods complement each other to a certain degree. Whereas EIT signals are relatively large at low ion content, magnetic flux densities can be best observed for large electrical conductivities. Understanding the basic processes connected with water movement in the pore space in the laboratory will improve the understanding of water fluxes on a larger scale. The most important results of this work are concerning the inhomogeneous distribution of salt caused by evaporation and the performance of MMR imaging for detecting small quantities of highly conducting fluids in a matrix of low conductivity and vice versa. The latter results are particularly interesting for monitoring at saline or dry sites which are investigated for nuclear waste disposal.

\section{Acknowledgement}

Katrin Breede was supported by the SFB TR32 "Patterns in soil-vegetation-atmosphere systems: monitoring, modeling and data assimilation" which is sponsored by the DFG. 


\section{References}

Bodvarsson, G.S., Boyle, W., Patterson, R. and Williams, D., 1999. Overview of scientific investigations at Yucca Mountain - the potential repository for high-level nuclear waste. J. Contam. Hydrol. 38(1-3), 3-24.

Cosenza, P., Ghorbani, A., Florsch, N. and Revil, A., 2007. Effects of drying on the lowfrequency electrical properties of Tournemire Argillites. Pure Appl. Geophys. 164(10), 20432066.

deGroot-Hedlin, C. and Constable, S., 1990. Occam's inversion to generate smooth, twodimensional models from magnetotelluric data. Geophysics 55(12), 1613-1624.

Edwards, R.N., Nabighian, M.N. and Aston, M.W., 1991. The magnetometric resistivity method, in M.N. Nabighian, (ed.), Investigations in Geophysics no. 3: Electromagnetic Methods in Applied Geophysics, Vol. 2, Applications, Society of Exploration Geophysicists, Tulsa, USA, pp. 47-104.

Giese, R., Henninges, J., Lüth, S., Morozova, D., Schmidt-Hattenberger, C., Würdemann, H., Zimmer, M., Cosma, C., Juhlin, C., and CO2SINK Group, 2009. Monitoring at the $\mathrm{CO}_{2} \mathrm{SINK}$ site: A concept integrating geophysics, geochemistry and microbiology. Energy Procedia 1(1), 2251-2259.

Jockwer, N., Wieczorek, K. and Fernández, A.M., 2007. Measurement of gas generation, water content and change in the water distribution in a heater experiment in the underground laboratory Mont Terri. Phys. Chem. Earth 32(1-7), 530-537. 
Kemna, A., Tillmann, A. Verweerd, A., Zimmermann, E. and Vereecken, H., 2003. MERIT a new magneto-electrical resistivity imaging technique: Part 1) modeling and tomographic reconstruction, Proceedings of the $3^{\text {rd }}$ World Congress on Industrial Process Tomography, Banff, Canada, pp. 256-261.

Kessels, W., Flentge, I. and Kolditz, H., 1985. DC geoelectric sounding to determine water content in the salt mine Asse (FRG). Geophys. Prospect. 33(3), 436-446.

Koestel, J., Kemna, A., Javaux, M., Binley, A., and Vereecken, H., 2008. Quantitative imaging of solute transport in an unsaturated and undisturbed soil monolith with 3-D ERT and TDR. Water Resour. Res. 44(12), W12411, 1-17.

Koestel, J., Vanderborght, J., Javaux, M., Kemna, A., Binley, A., and Vereecken, H., 2009. Noninvasive 3-D transport characterization in a sandy soil using ERT: 2. Transport processes inference. Vadose Zone J. 8(3), 723-734.

Kruschwitz, S. and Yaramanci, U., 2004. Detection and characterization of the disturbed rock zone in claystone with the complex resistivity method. J. Appl. Geophys. 57(1), 63-79.

Kulenkampff, J.M. and Yaramanci, U., 1993. Frequency-dependent complex resistivity of rock-salt samples and related petrophysical parameters. Geophys. Prospect. 41(8), 995-1008.

Kwon Y.W. and Bang, H.; 1997. The Finite Element Method using MATLAB, CRC Press, Boca Raton, FL. 
Müller, K., Vanderborght, J., Englert, A., Kemna, A., Huisman, J.A., Rings, J. and Vereecken, H., 2010. Imaging and characterization of solute transport during two tracer tests in a shallow aquifer using electrical resistivity tomography and multilevel groundwater samplers. Water Resour. Res. 46(3), W03502, 1-23.

Nabighian, M.N., 1988. Investigations in Geophysics no. 3: Electromagnetic Methods in Applied Geophysics, vol. 1, Theory, SEG, Tulsa, Oklahoma.

Nabighian, M.N., 1991. Investigations in Geophysics no. 3: Electromagnetic Methods in Applied Geophysics, vol. 2, Applications, Part A and B, SEG, Tulsa, Oklahoma..

Roberts, J.J. and Lin, W., 1997. Electrical properties of partially saturated Topopah Spring tuff: Water distribution as a function of saturation. Water Resour. Res. 33(4), 577-587.

Rubin, Y. and Hubbard, S., 2005. Water Science and Technology Library, vol. 50, Hydrogeophysics, Springer, Dordrecht, The Netherlands.

Shokri, N., Lehmann, P. and Or, D., 2009. Characteristics of evaporation from partially wettable porous media. Water Resour. Res. 45, W02415, 1-12.

Sumerling, T. and Smith, P., 1998. Disposal of nuclear fuel waste - Geological alternatives, designs, implementation, and long term safety. Interdiscip. Sci. Rev. 23(3), 214-232.

Titov, K., Kemna, A., Tarasov, A. and Vereecken, H., 2004. Induced polarization of unsaturated sands determined through time domain measurements. Vadose Zone J. 3(4), $1160-1168$. 
Ulrich C. and Slater, L.D., 2004. Induced polarization measurements on unsaturated, unconsolidated sands. Geophysics 69(3), 762-771.

Vereecken, H., Huisman, J.A., Bogena, H., Vanderborght, J., Vrugt, J.A. and Hopmans, J.W., 2008. On the value of soil moisture measurements in vadose zone hydrology: A review. Water Resour. Res. 44, W00D06, 1-21.

Verweerd, A.J., 2007. Performance Analysis and Characterisation of a new MagnetoElectrical Measurement System for Electrical Conductivity Imaging. Dissertation, University of Bonn, Schriften des Forschungszentrums Jülich . Reihe Umwelt / Environment; 75, Jülich, Forschungszentrum, Zentralbibliothek.

Xu, G., Wu, H., Yang, S., Liu, S., Li, Y., Yang, Q., Yan, W. and Wang, M., 2005. 3D electrical impedance tomography forward problem with a finite element method. IEEE Trans. Magn. 41(5), 1832-1835.

Yaramanci, U. and Flach, D., 1992. Resistivity of rock-salt in Asse (Germany) and petrophysical aspects. Geophys. Prospect. 40(1), 85-100.

Yaramanci, U., 1994. Relation of in situ resistivity to water content in salt rocks. Geophys. Prospect. 42(3), 229-239.

Yaramanci, U., 2000. Geoelectric exploration and monitoring in rock salt for the safety assessment of underground waste disposal sites. J. Appl. Geophys. 44(2-3), 181-196. 
Zimmermann, E., Verweerd, A., Glaas, W., Tillmann, A. and Kemna A., 2005, An AMR sensor-based measurement system for magnetoelectrical resistivity tomography. IEEE Sens. J. $5(2), 233-241$.

Zimmermann, E., Kemna, A., Berwix, J., Glaas, W., Münch, H.M. and Huisman, J.A., 2008ª . A high-accuracy impedance spectrometer for measuring sediments with low polarizability. Meas. Sci. Technol. 19(10), 105603, 1-9.

Zimmermann, E., Kemna, A., Berwix, J., Glaas, W., and Vereecken, H., 2008 . EIT measurement system with high phase accuracy for the imaging of spectral induced polarization properties of soils and sediments. Meas. Sci. Technol. 19(9), 094010, 1-9. 


\section{Nomenclature}

$\mathrm{B}=$ magnetic flux density $[\mathrm{T}]$

$\mathrm{I}=$ current $[\mathrm{A}]$

$\mathrm{J}=$ current density $\left[\mathrm{A} \mathrm{m}^{-2}\right]$

$\mathrm{r}=$ position with respect to the central axis [m]

$\mathbf{r}=$ position vector, in particular position vector of sensor $[\mathrm{m}]$

$\mathbf{r}^{\prime}=$ position vector of volume element $[\mathrm{m}]$

$S^{\prime}=$ normalized real part of complex conductivity $=\sigma^{\prime} / \sigma_{0}$ '

$\mathrm{S}^{\prime \prime}=$ normalized imaginary part of complex conductivity $=\sigma ' \sigma_{0}$,

$\mathrm{S}_{\mathrm{w}}=$ water saturation $=$ relative volumetric water content related to complete saturation

$\mathrm{t}=$ time in days $[\mathrm{d}]$

$\mathrm{v}=$ volume $\left[\mathrm{m}^{3}\right]$

$\mathrm{V}=$ electrical potential $[\mathrm{m}]$

$\mathrm{x}, \mathrm{y}, \mathrm{z}=$ coordinates $[\mathrm{m}]$

$\delta=$ Dirac delta function

$\mu=$ magnetic permeability $\left[\mathrm{V} \mathrm{s} \mathrm{A} \mathrm{A}^{-1}\right.$ ]

$\mu_{0}=$ magnetic constant $=1.257 \cdot 10^{-6} \mathrm{~V} \mathrm{~s} \mathrm{~A}^{-1} \mathrm{~m}^{-1}$

$\sigma=$ absolute value of complex electrical conductivity $\left[\mathrm{S} \mathrm{m}^{-1}\right]$

$\sigma^{*}=$ complex electrical conductivity $\left[\mathrm{S} \mathrm{m}^{-1}\right]$

$\sigma^{\prime}=$ real part of complex electrical conductivity $\left[\mathrm{S} \mathrm{m}^{-1}\right]$

$\sigma_{0}{ }^{\prime}=$ imaginary part of complex electrical conductivity for complete water saturation $\left[\mathrm{S} \mathrm{m}^{-1}\right]$

$\sigma "=$ imaginary part of complex electrical conductivity $\left[\mathrm{S} \mathrm{m}^{-1}\right]$

$\sigma_{0} "=$ imaginary part of complex electrical conductivity for complete water saturation $\left[\mathrm{S} \mathrm{m}^{-1}\right]$

$\nabla^{\prime}=$ Nabla operator operative at position $\mathrm{r}^{\prime}$ 
Figures

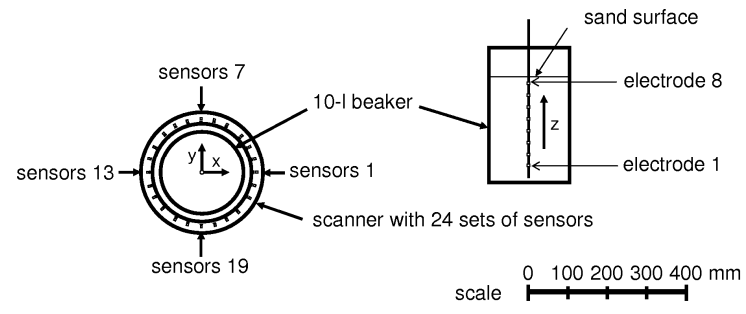

Figure 1: Schematic representation of the MERIT experiment

a)

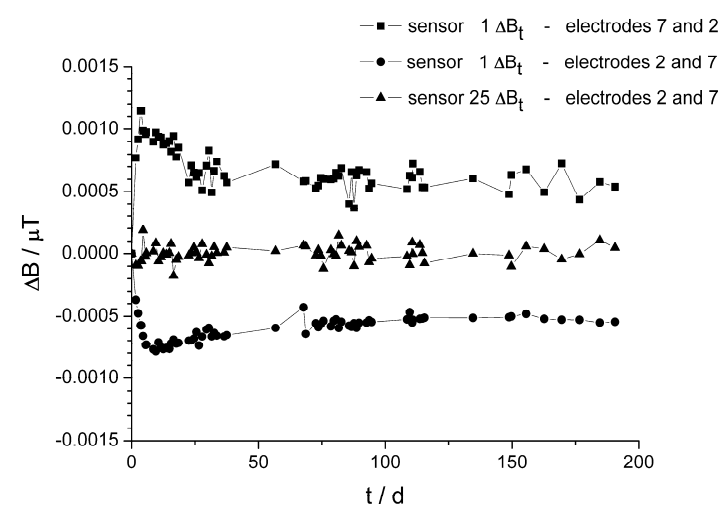

b)

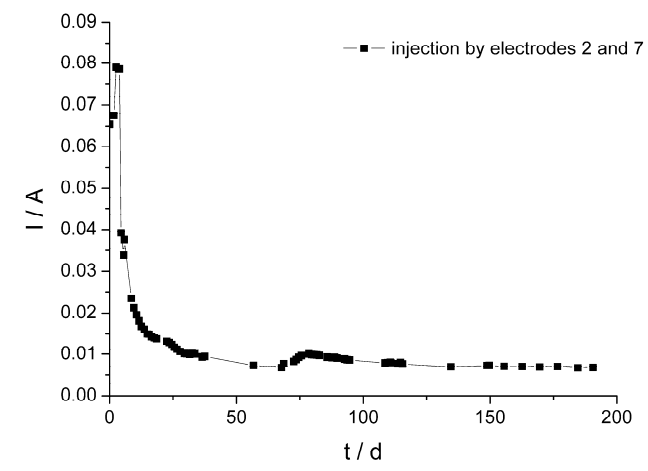

Figure 2: (a) Magnetic flux density and (b) current during evaporation 

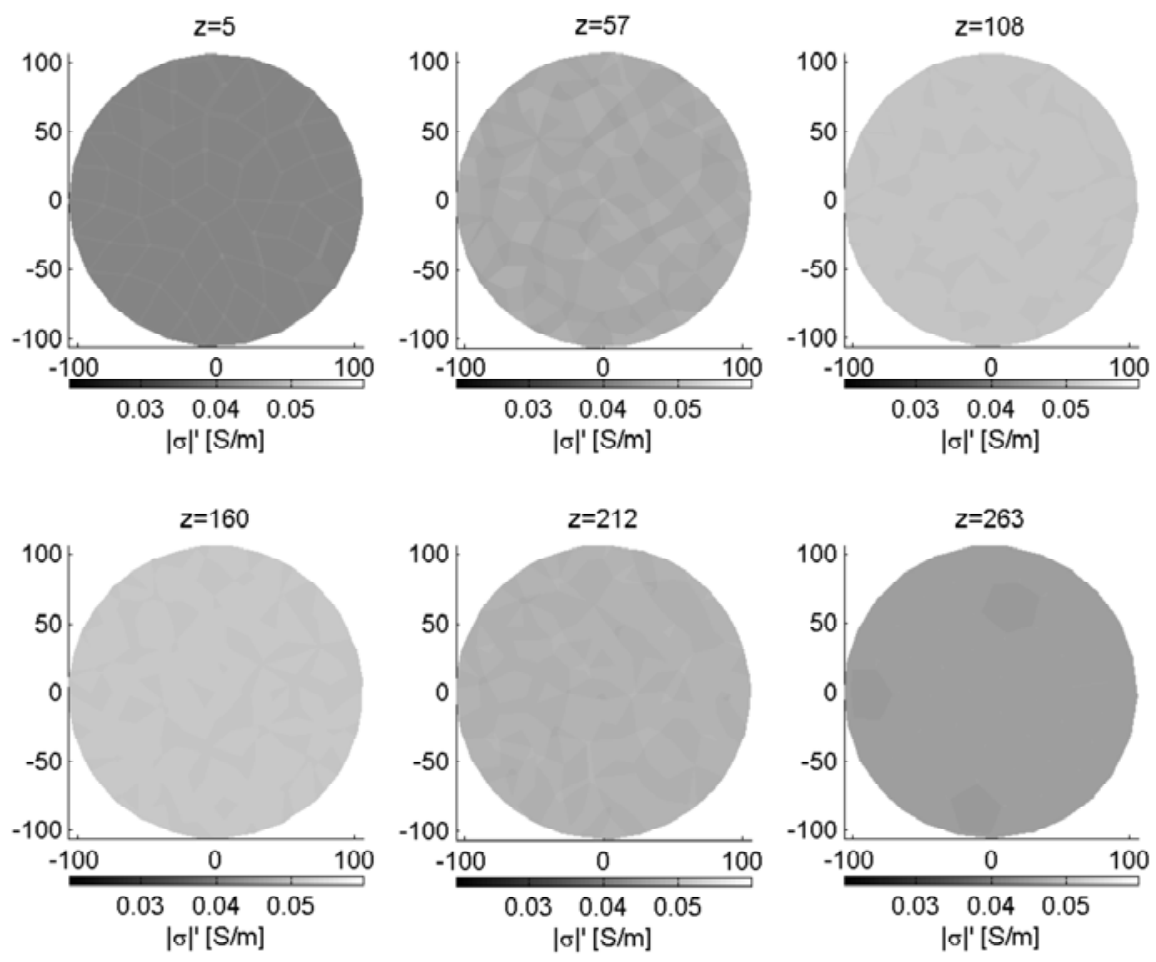

Figure 3: Images of 1D electrical conductivity distribution (cuts at different heights $\mathrm{z}[\mathrm{mm}]$ ) for saturated sand before the evaporation experiment with MERIT 
a)
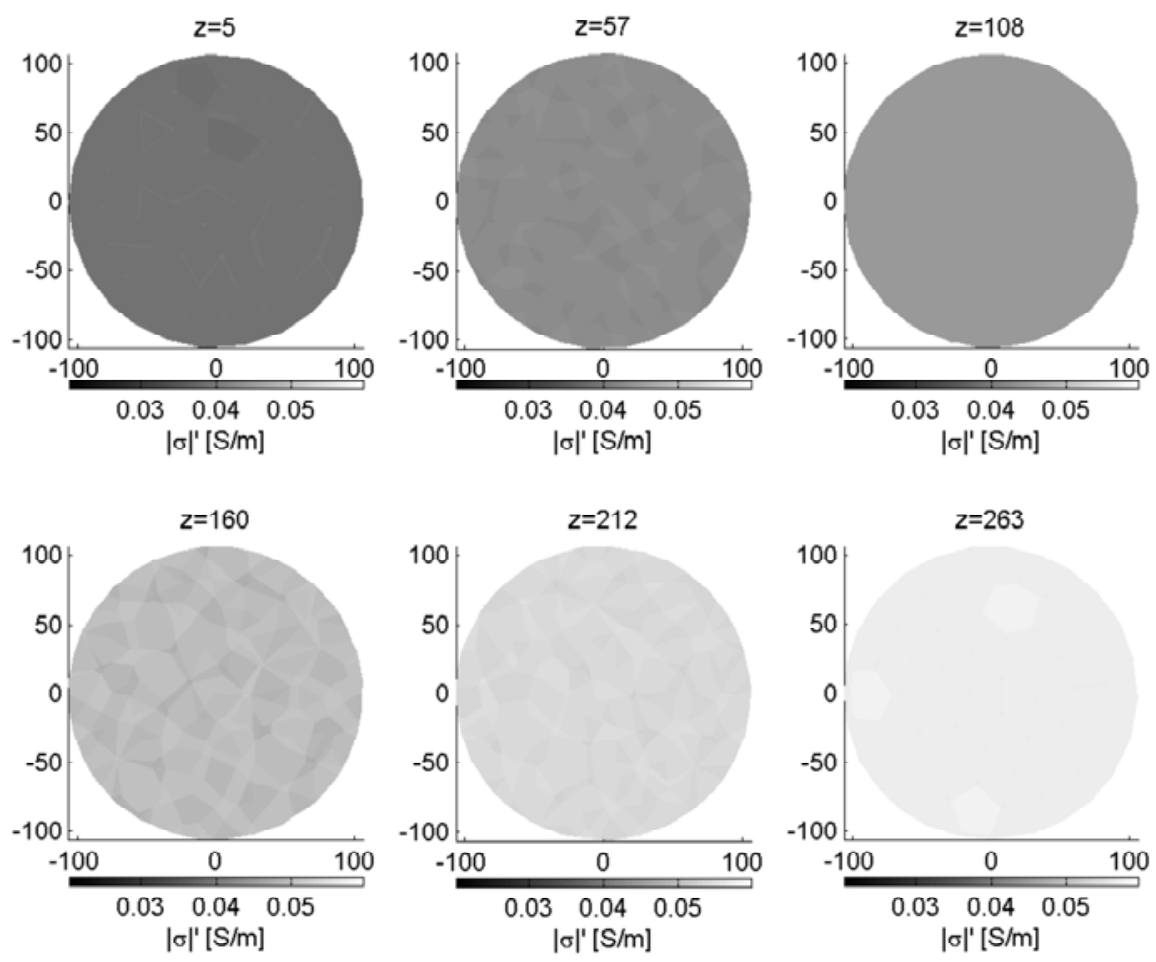

b)
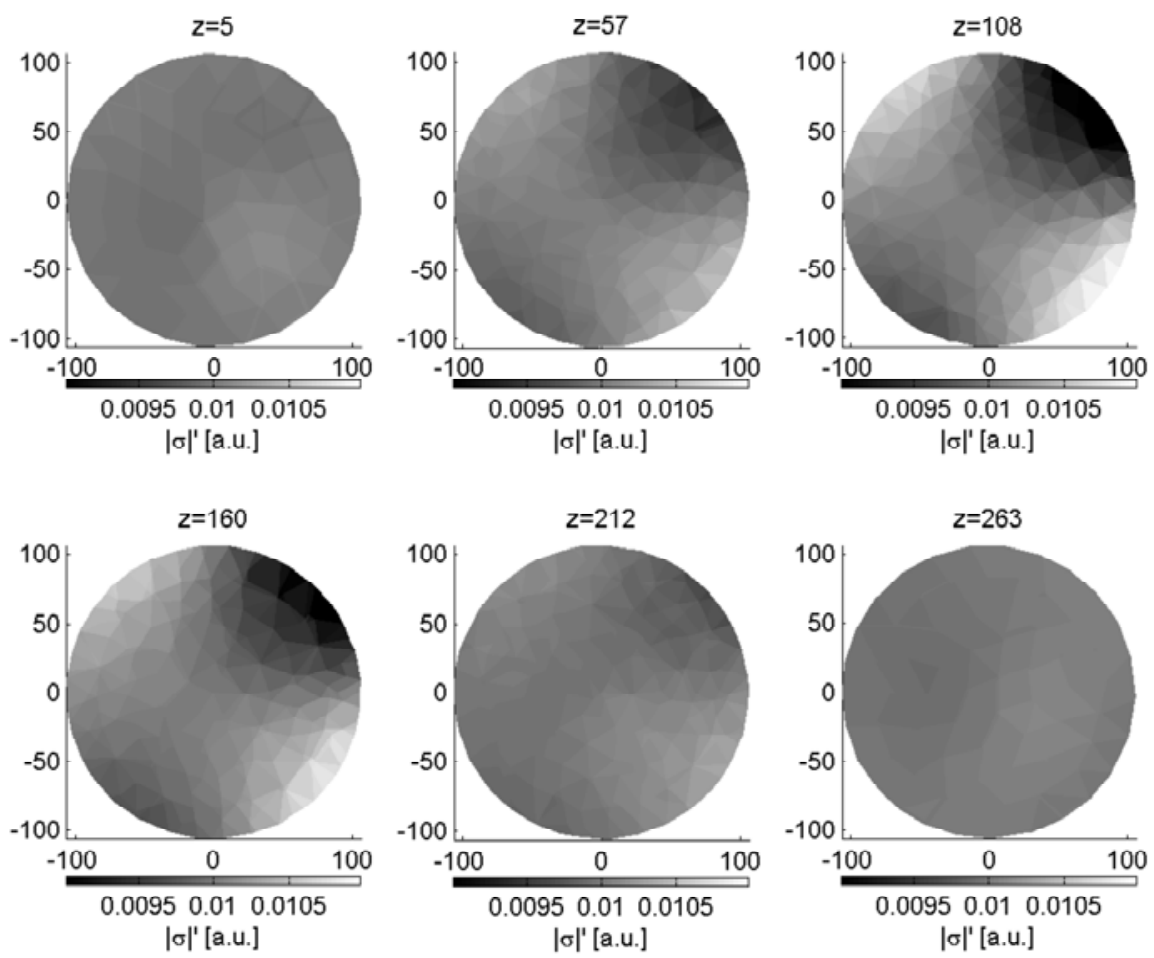

Figure 4: MERIT results (cuts at different heights $\mathrm{z}[\mathrm{mm}]$ ) after the infiltration of a negative tracer

a) Images from $1 \mathrm{D}$ electrical conductivity distribution

b) Images of relative electrical conductivities in arbitrary units calculated with difference inversion of normalized magnetic flux densities 
a)
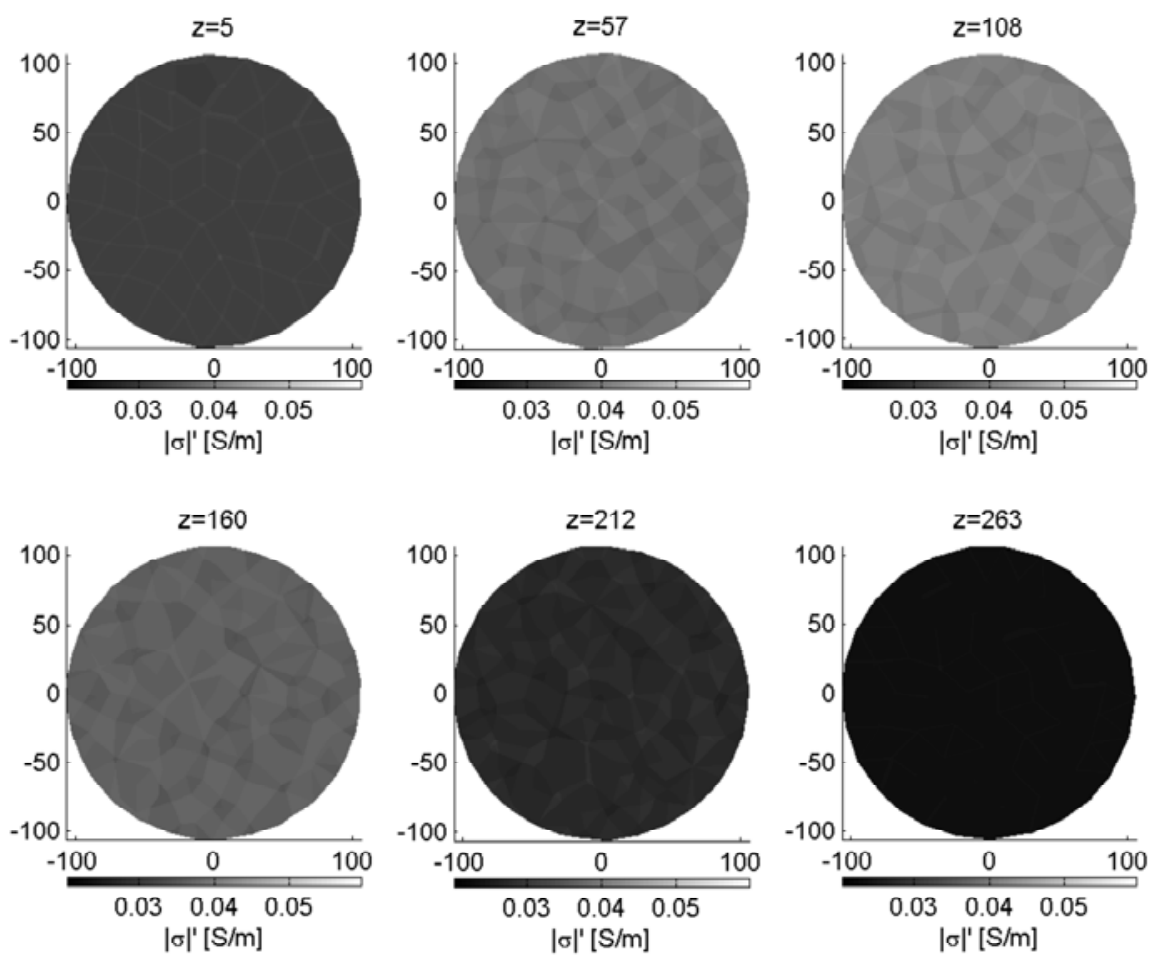

b)
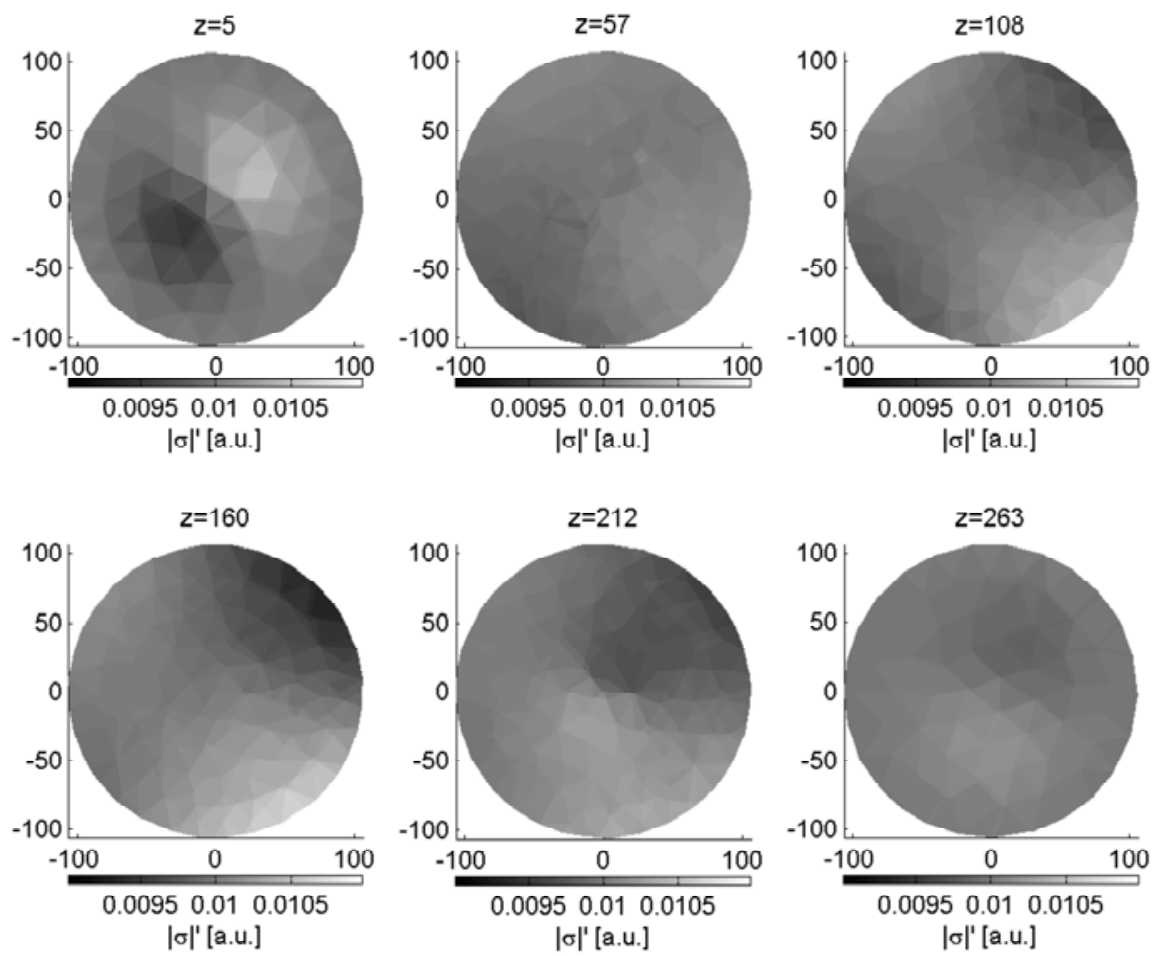

Figure 5: MERIT results (cuts at different heights $\mathrm{z}[\mathrm{mm}]$ ) after 9 days of evaporation a) Images from $1 \mathrm{D}$ electrical conductivity distribution

b) Images of relative electrical conductivities in arbitrary units calculated with difference inversion of normalized magnetic flux densities 

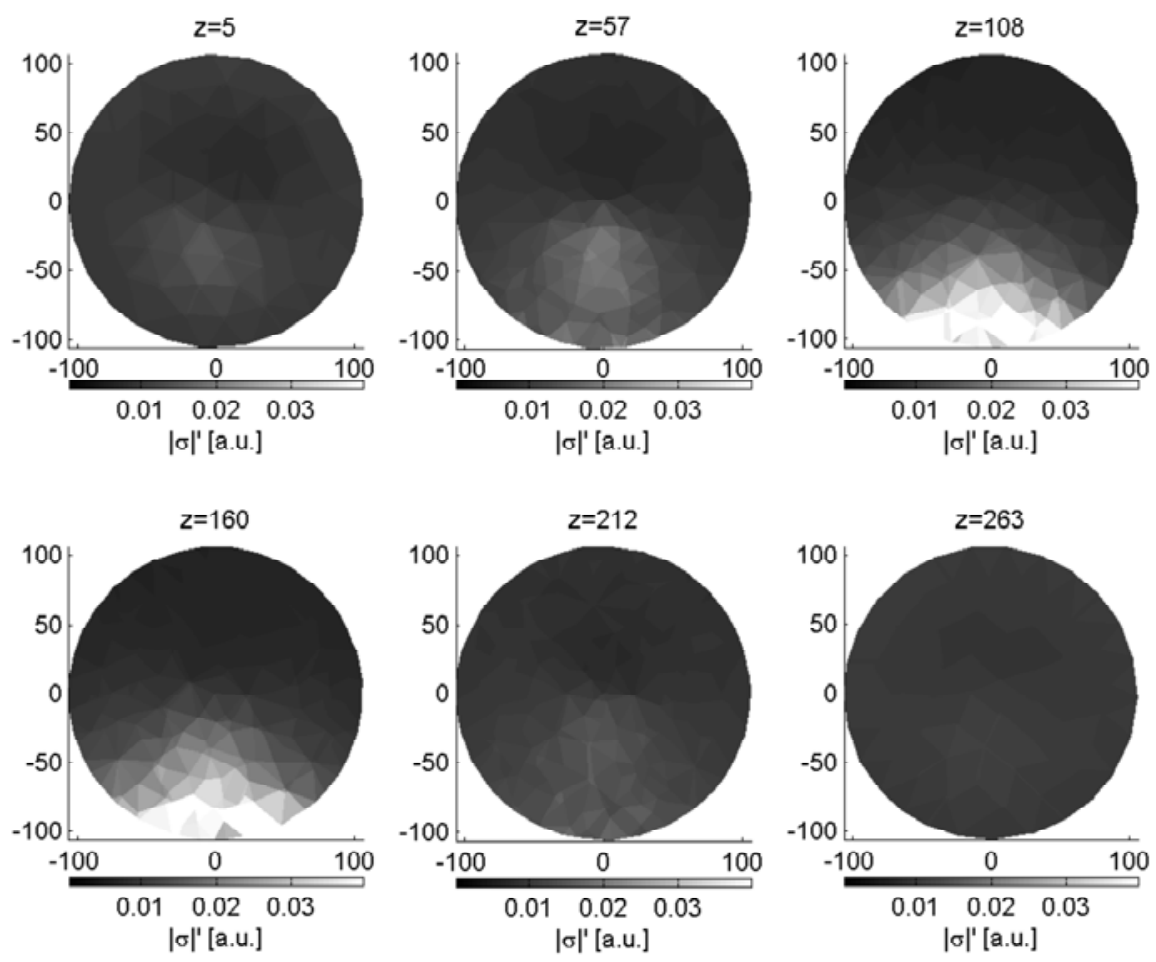

Figure 6: Images of relative electrical conductivities in arbitrary units calculated with difference inversion of normalized magnetic flux densities (cuts at different heights $\mathrm{z}[\mathrm{mm}]$ ) after the infiltration of a salt tracer into dry sand

a)

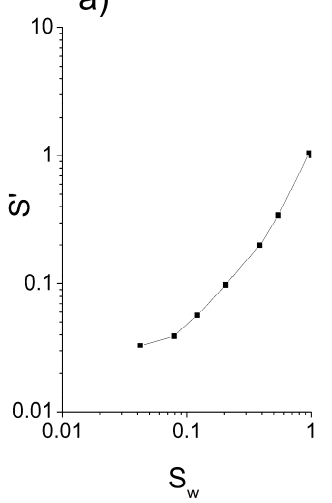

b)

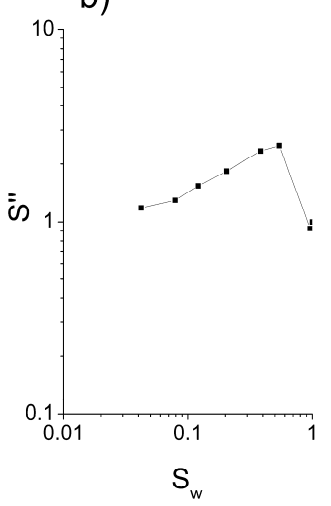

Figure 7: (a) Real part of normalized electrical conductivity and (b) imaginary part of normalized electrical conductivity as a function of water saturation 

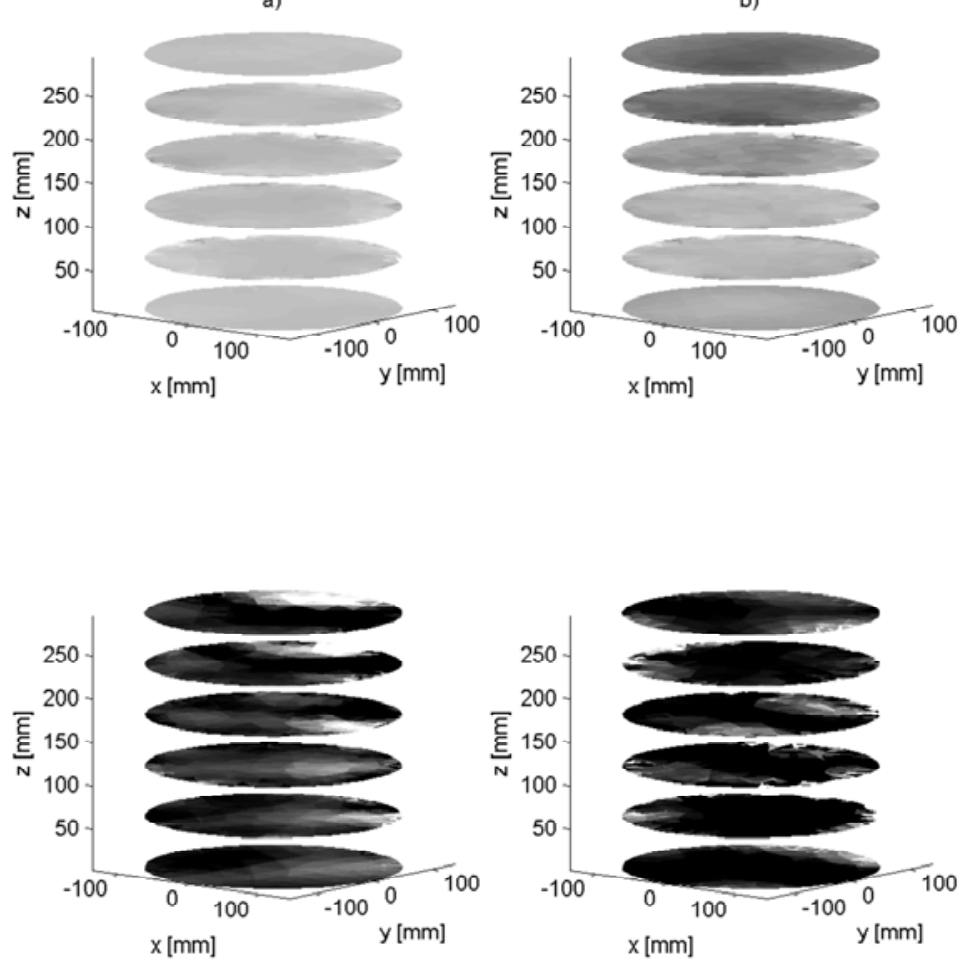

b)
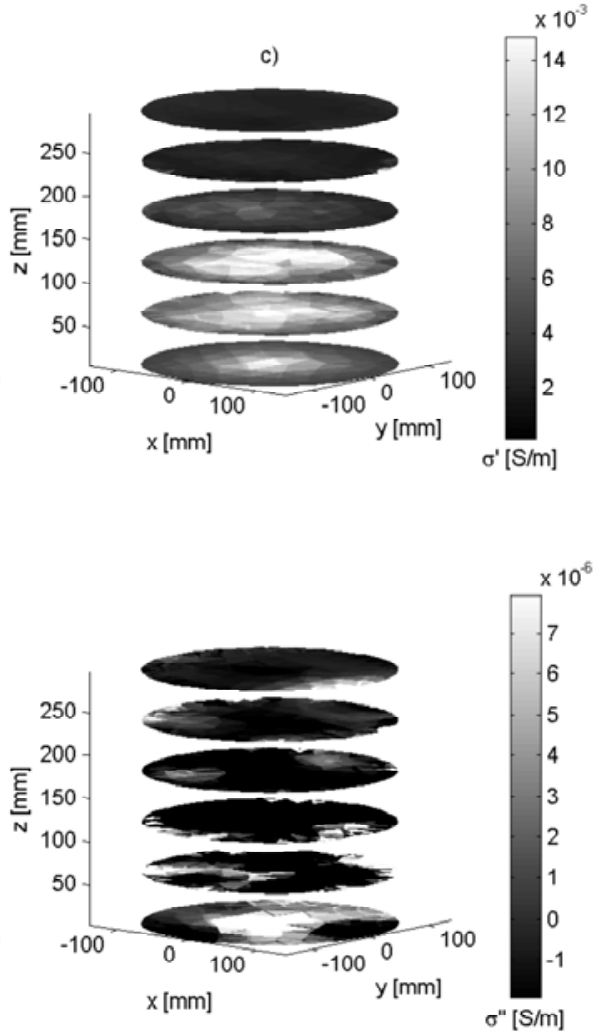

Figure 8: Distribution of the real part $\sigma^{\prime}$ and imaginary part $\sigma^{\prime \prime}$ of complex electrical conductivity after 0 (a), 7 (b), and 21 (c) days of evaporation

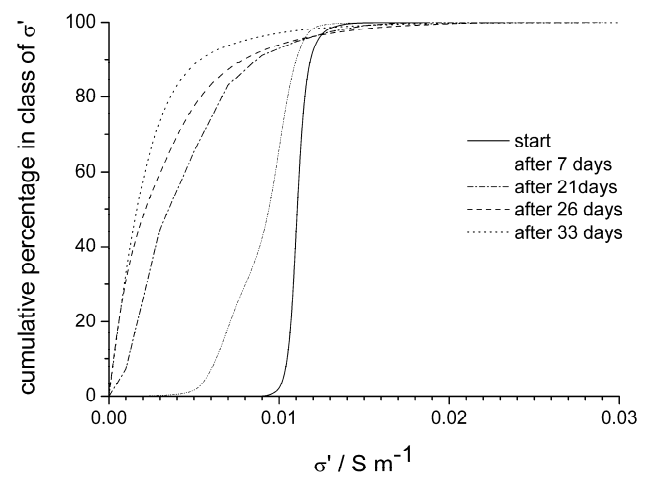

Figure 9: Frequency of voxels as a function of $\sigma^{\prime}$ during the EIT evaporation experiment 
a)

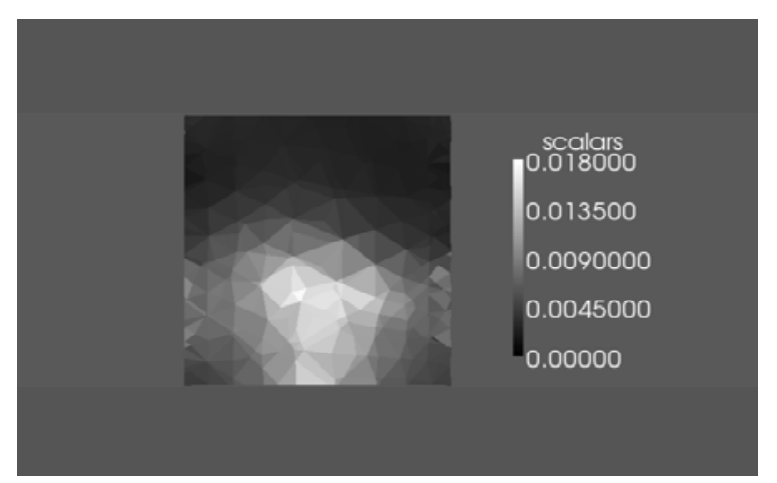

b)

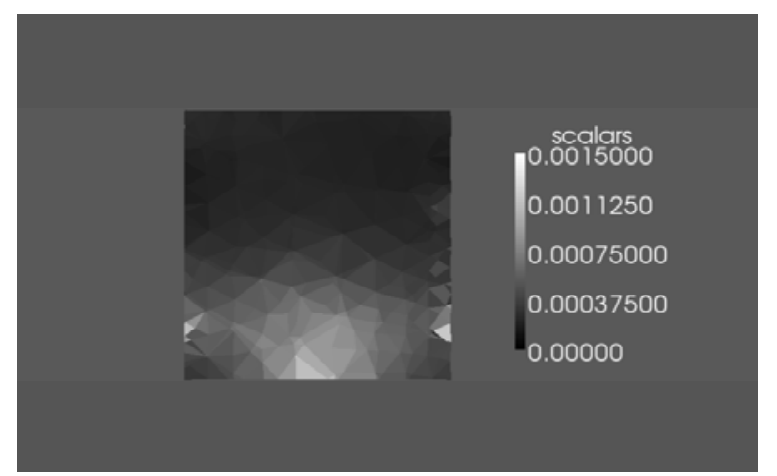

Figure 10: Formation of a core of enhanced $\sigma^{\prime}\left[\mathrm{S} \mathrm{m}^{-1}\right]$ during the EIT evaporation experiment after 21 days (a) and 143 days (cut through the xz-plane) 